\title{
Sampling theorems for shift-invariant spaces, Gabor frames, and totally positive functions
}

\author{
Karlheinz Gröchenig ${ }^{1}$ • José Luis Romero ${ }^{1,3}$. \\ Joachim Stöckler ${ }^{2}$
}

Received: 12 December 2016 / Accepted: 23 September 2017 /

Published online: 20 October 2017

(C) The Author(s) 2017. This article is an open access publication

\begin{abstract}
We study nonuniform sampling in shift-invariant spaces and the construction of Gabor frames with respect to the class of totally positive functions whose Fourier transform factors as $\hat{g}(\xi)=\prod_{j=1}^{n}\left(1+2 \pi i \delta_{j} \xi\right)^{-1} e^{-c \xi^{2}}$ for $\delta_{1}, \ldots, \delta_{n} \in \mathbb{R}, c>0$ (in which case $g$ is called totally positive of Gaussian type). In analogy to Beurling's sampling theorem for the Paley-Wiener space of entire functions, we prove that every separated set with lower Beurling density $>1$ is a sampling set for the shift-invariant space generated by such a $g$. In view of the known necessary density conditions, this result is optimal and validates the heuristic reasonings in the engineering literature. Using a
\end{abstract}

K. G. was supported in part by the Project P26273 - N25 of the Austrian Science Fund (FWF). J. L. R. gratefully acknowledges support from the Austrian Science Fund (FWF): P 29462 N35.

$凶$ Karlheinz Gröchenig

karlheinz.groechenig@univie.ac.at

José Luis Romero

jose.luis.romero@univie.ac.at

Joachim Stöckler

joachim.stoeckler@math.tu-dortmund.de

1 Faculty of Mathematics, University of Vienna, Oskar-Morgenstern-Platz 1, 1090 Vienna, Austria

2 Faculty of Mathematics, TU Dortmund, Vogelpothsweg 87, 44221 Dortmund, Germany

3 Acoustics Research Institute, Austrian Academy of Sciences, Wohllebengasse 12-14, 1040 Vienna, Austria 
subtle connection between sampling in shift-invariant spaces and the theory of Gabor frames, we show that the set of phase-space shifts of $g$ with respect to a rectangular lattice $\alpha \mathbb{Z} \times \beta \mathbb{Z}$ forms a frame, if and only if $\alpha \beta<1$. This solves an open problem going back to Daubechies in 1990 for the class of totally positive functions of Gaussian type. The proof strategy involves the connection between sampling in shift-invariant spaces and Gabor frames, a new characterization of sampling sets "without inequalities" in the style of Beurling, new properties of totally positive functions, and the interplay between zero sets of functions in a shift-invariant space and functions in the Bargmann-Fock space.

Mathematics Subject Classification 42C15 - 42C40 - 94A20

\section{Introduction}

\subsection{Nonuniform sampling}

Beurling's sampling theory for the Paley-Wiener space is at the crossroad of complex analysis and signal processing and has served as a model and inspiration for many generations of sampling theorems in both fields. To recapitulate Beurling's results, let

$$
\mathrm{PW}^{2}=\left\{f \in L^{2}(\mathbb{R}): \operatorname{supp} \hat{f} \subseteq[-1 / 2,1 / 2]\right\}
$$

be the Paley-Wiener space, where $\hat{f}(\xi):=\int_{\mathbb{R}} f(x) e^{-2 \pi i x \xi} d x$ is the Fourier transform. It consists of restrictions of entire functions of exponential type to the real line and is called the space of bandlimited functions by engineers and applied mathematicians. This space was and still is the prevailing signal model for phenomena as diverse as radio signals, temperatures and biomedical measurements [60].

A separated set $\Lambda \subseteq \mathbb{R}$ is called a sampling set for $\mathrm{PW}^{2}$, if there exist $A, B>0$ such that

$$
A\|f\|_{2}^{2} \leq \sum_{\lambda \in \Lambda}|f(\lambda)|^{2} \leq B\|f\|_{2}^{2} \quad \text { for all } f \in \mathrm{PW}^{2} .
$$

The understanding of sampling sets may be considered a core problem in complex analysis, but is also a fundamental problem in signal processing. Since a signal is always sampled and then processed, sampling is a key operation in the analog-digital conversion. The sampling inequality (1.1) guarantees that the samples carry the complete information about the signal and that the signal can be recovered from the samples with some stability with respect to measurement and processing errors. 
Beurling $[10,11]$ characterized sampling sets by means of their density. The (lower) Beurling density is the average density of samples per unit and is formally defined as

$$
D^{-}(\Lambda):=\liminf _{R \rightarrow \infty} \inf _{x \in \mathbb{R}} \frac{\#(\Lambda \cap[x-R, x+R])}{2 R} .
$$

The theorems of Beurling give an almost complete characterization of sampling sets for the Paley-Wiener space $\mathrm{PW}^{2}$.

Theorem 1.1 (a) If $\Lambda \subseteq \mathbb{R}$ is separated and $D^{-}(\Lambda)>1$, then $\Lambda$ is a sampling set for $\mathrm{PW}^{2}$.

(b) Conversely, if $\Lambda$ is a sampling set for $\mathrm{PW}^{2}$, then $D^{-}(\Lambda) \geq 1$.

A related result with uniform densities occurs in the work of Duffin and Schaeffer [18]. The case of critical density $D^{-}(\Lambda)=1$ is delicate and was settled in [49]. Subsequently, the necessary condition (b) above has been extended and adapted to many different situations, notably to Paley-Wiener spaces in higher dimension with respect to general spectra by Landau [43], and to sampling in spaces of analytic functions [57]. The necessary conditions for sampling can even be formulated and proved for sampling in reproducing kernel Hilbert spaces over a metric measure space [5,22].

The sufficient condition (a) is much deeper and more subtle and has been extended only to a handful of situations, notably to sampling in the BargmannFock space of entire functions by Lyubarskii and Seip $[47,56]$. For bandlimited functions with general spectra, even the existence of sets that are both sampling and interpolating is a very challenging problem $[42,45]$.

In this article we develop an analog of Beurling's theory in an unexpected direction beyond classical complex analysis and study sampling in shiftinvariant spaces. This notion comes from approximation theory and replaces the Paley-Wiener space in many modern applications because often data acquisition demands a more flexible setting, where signals are not strictly bandlimited, but only approximately so. For the definition, let $g \in L^{2}(\mathbb{R})$ be a generating function and consider the space

$$
V^{2}(g)=\left\{f \in L^{2}(\mathbb{R}): f=\sum_{k \in \mathbb{Z}} c_{k} g(\cdot-k), c \in \ell^{2}(\mathbb{Z})\right\} .
$$

We always assume that $g$ possesses stable translates so that $\|f\|_{2} \asymp\|c\|_{2}$. Typically the Fourier transform of $g$ decays rapidly outside an interval and thus functions in $V^{2}(g)$ are approximately bandlimited. In the extreme case of $\hat{g}=\chi_{[-1 / 2,1 / 2]}$, the shift-invariant space $V^{2}(g)$ is precisely the Paley-Wiener space $\mathrm{PW}^{2}$. Intuitively, functions in a general shift-invariant space $V^{2}(g)$ have a rate of innovation of one degree per space unit $[3,12,60]$. It is therefore 
expected that nonuniform sampling theorems similar to Theorem 1.1 hold for general shift-invariant spaces. Such claims are usually backed by a heuristic comparison to the bandlimited case, but, in spite of their central role in signal processing, they have been given only moderate formal support so far. In [2] we suggested that every reasonable generator $g$ comes with a notion of a suitable $g$-dependent density $D_{g}^{-}(\Lambda)$, such that Beurling's formulation carries over verbatim. Despite many efforts and dozens of publications this suggestion and the attached conjectures remained untouched.

The existing results for sampling in a shift-invariant space use the covering density $\delta=2 \sup _{x \in \mathbb{R}} \inf _{\lambda \in \Lambda}|x-\lambda|$ instead of the Beurling density. Typical statements assert that every set $\Lambda$ with a "sufficiently small" covering density $\delta$ is a sampling set for $V^{2}(g)$. However, because of the unspecified (and usually large) gap between the known necessary density and the proven sufficient density these results are far from optimal and lack practical relevance. See [3] for a survey of sampling in shift-invariant spaces including many qualitative results. Sharp results in terms of the covering density (" $\delta<1$ is sufficient for sampling") are known only when the generator $g$ is a B-spline [2], an exponential B-spline [41], or a totally positive function of finite type [29].

Our objective is to develop a sampling theory in a large class of shiftinvariant spaces in the style of Beurling and to prove the best possible results. As a natural and suitable class of generators we deal with totally positive functions. A function $g \in L^{1}(\mathbb{R})$ is called totally positive, if for all $N \in \mathbb{N}$ and real numbers $x_{1}<x_{2}<\cdots<x_{N}, y_{1}<y_{2}<\cdots<y_{N}$ the matrix

$$
\left(g\left(x_{j}-y_{k}\right)\right)_{j, k=1, \ldots, N}
$$

has non-negative determinant. This property is fundamental in statistics, approximation theory, and data interpolation [40]. In the following we will deal with a subset of totally positive functions and call a function $g$ on $\mathbb{R}$ totally positive of Gaussian type if its Fourier transform factors as

$$
\hat{g}(\xi)=\prod_{j=1}^{n}\left(1+2 \pi i \delta_{j} \xi\right)^{-1} e^{-c \xi^{2}}, \quad \delta_{1}, \ldots, \delta_{n} \in \mathbb{R}, c>0, \quad n \in \mathbb{N} \cup\{0\} .
$$

By Schoenberg's characterization [52,54] of totally positive functions, every $g$ satisfying (1.3) is totally positive.

Our first main result is the following (almost) characterization of sampling sets in $V^{2}(g)$ in analogy to Beurling's theorem.

Theorem 1.2 Assume that $g$ is a totally positive function of Gaussian type as in (1.3). If $\Lambda \subseteq \mathbb{R}$ is separated and $D^{-}(\Lambda)>1$, then $\Lambda$ is a sampling set for $V^{2}(g)$. 
Since a sampling set for $V^{2}(g)$ must necessarily satisfy $D^{-}(\Lambda) \geq 1$ by [2,5], this result is optimal. Theorem 1.2 thus validates the heuristic understanding of the signal processing community that nonuniform sampling above the critical density (the Nyquist rate) leads to stable reconstruction in a shift-invariant space. As with bandlimited functions [49], the case of the critical density $D^{-}(\Lambda)=1$ is subtle and deserves a more detailed analysis. Generically, the set $\Lambda=r+\mathbb{Z}$ is a sampling set, but there always exists at least one $r_{0} \in[0,1]$ such that $r_{0}+\mathbb{Z}$ fails to be a sampling set.

To the best of our knowledge, Theorem 1.2 is the first result of this kind beyond a purely analytic setting (although complex variable methods enter our proofs through a backdoor). In contrast to the covering density, Theorem 1.2 allows for truly nonuniform sets with large gaps and underscores the use of Beurling's density as the right performance metric for sampling.

We stress that Theorem 1.2 is new even for the shift-invariant space with Gaussian generator. We believe that it opens a new avenue in approximation theory of radial basis functions, because sampling inequalities have become an integral part of the error analysis for scattered data interpolation [30,48]. We also have a dual result for interpolation in shift-invariant spaces, formulated in terms of Beurling's upper density $D^{+}(\Lambda)$.

Theorem 1.3 Let $\varphi(x)=e^{-c x^{2}}$ be a Gaussian. If $\Lambda \subseteq \mathbb{R}$ is separated and $D^{+}(\Lambda)<1$, then $\Lambda$ is an interpolating set for $V^{2}(\varphi)$. This means that for all $a \in \ell^{2}(\Lambda)$ there exists $f \in V^{2}(\varphi)$ such that $f(\lambda)=a_{\lambda}$.

\subsection{Gabor analysis}

Surprisingly, the problem of sampling in shift-invariant spaces is intimately connected with the construction of Gabor frames. As a consequence of Theorem 1.2 we make substantial progress towards a conjecture that was originally expressed by I. Daubechies in [15] and then refined in [26,29].

Let $(x, \xi) \in \mathbb{R}^{2}$ and $M_{\xi} T_{x} g(t)=e^{2 \pi i \xi t} g(t-x)$ be the corresponding phase-space shift (time-frequency shift). For a separated set $\Omega \subseteq \mathbb{R}^{2}$ and a generator $g \in L^{2}(\mathbb{R})$ (a window function in the established terminology), let $\mathcal{G}(g, \Omega)=\left\{M_{\nu} T_{\mu} g:(\mu, \nu) \in \Omega\right\}$ be the set of time-frequency shifts over $\Omega$. We study the question when $\mathcal{G}(g, \Omega)$ is a Gabor frame, i.e., when there exist $A, B>0$, such that

$$
A\|f\|_{2}^{2} \leq \sum_{(\mu, \nu) \in \Omega}\left|\left\langle f, M_{\nu} T_{\mu} g\right\rangle\right|^{2} \leq B\|f\|_{2}^{2} \quad \text { for all } f \in L^{2}(\mathbb{R}) .
$$

In other words, $\Omega$ is a sampling set for the space $V=\left\{F \in L^{2}\left(\mathbb{R}^{2}\right)\right.$ : $F(x, \xi)=\left\langle f, M_{\xi} T_{x} g\right\rangle$ for $\left.f \in L^{2}(\mathbb{R})\right\}$. Again there is a universal necessary density condition given by the density theorem for Gabor frames [32]. If 
$\mathcal{G}(g, \Omega)$ is a frame for $L^{2}(\mathbb{R})$, then $D_{2}^{-}(\Omega) \geq 1$ (where $D_{2}^{-}$is the Beurling density on $\mathbb{R}^{2}$ ). Under mild conditions on the window $g$, the inequality is strict $D_{2}^{-}(\Omega)>1[4,9,28]$.

On the other hand, the derivation of optimal sufficient conditions for (1.4) to hold is an old and difficult problem in analysis and is usually studied only for lattices $\Omega=\alpha \mathbb{Z} \times \beta \mathbb{Z}, \alpha, \beta>0$ or $\Omega=A \mathbb{Z}^{2}$ with $A \in \mathrm{GL}(2, \mathbb{R})[15,23]$.

Over a period of 25 years a complete characterization was obtained only for the Gaussian $[47,56]$, the truncated and the symmetric exponential functions [36,37], the hyperbolic secant [38], and for totally positive functions of finite type $\geq 2$ [29]. The fact that all these functions are totally positive led to the following conjecture: for a continuous function $g \in L^{1}(\mathbb{R})$ the set $\mathcal{G}(g, \alpha \mathbb{Z} \times \beta \mathbb{Z})$ is a frame, if and only if $\alpha \beta<1$.

Our second main result proves this conjecture for totally positive functions of Gaussian type and extends the characterization of Gabor frames to semiregular sets of the form $\Omega=\Lambda \times \mathbb{Z}$ for $\Lambda \subseteq \mathbb{R}$.

Theorem 1.4 Assume that $g$ is a totally positive function of Gaussian type. Let $\Lambda \subseteq \mathbb{R}$ be a separated set.

Then $\mathcal{G}(g, \Lambda \times \mathbb{Z})$ is a Gabor frame for $L^{2}(\mathbb{R})$, if and only if $D^{-}(\Lambda)>1$.

We formulate the case of rectangular lattices explicitly as a corollary.

Corollary 1.5 Assume that $g$ is a totally positive function of Gaussian type. Then $\mathcal{G}(g, \alpha \mathbb{Z} \times \beta \mathbb{Z})$ is a frame, if and only if $\alpha \beta<1$.

What is still missing to prove the full conjecture is the class of totally positive functions of infinite type, where $\hat{g}(\xi)=\prod_{j=1}^{\infty}\left(1+2 \pi i \delta_{j} \xi\right)^{-1} e^{-c \xi^{2}}$, $\delta_{j} \in \mathbb{R} \backslash\{0\}, c \geq 0$. It seems tempting to extend Corollary 1.5 by some form of limiting procedure, but so far we have not succeeded in finding a rigorous argument.

Let us emphasize that totally positive functions constitute the only class of window functions for which a characterization of all frame generating lattices seems within reach. For other natural classes of window functions, such as the $B$-splines or the Hermite functions, only partial results are known [27], and all explicit conjectures have been disproved [44] and need a thorough reformulation.

\subsection{Totally positive functions}

As an important tool, we will derive a new property of totally positive functions related to zero sets in shift-invariant spaces. The following result will serve as a central technical tool for the sampling results.

Theorem 1.6 Assume that $g$ is totally positive of Gaussian type. Let $f=$ $\sum_{k \in \mathbb{Z}} c_{k} g(\cdot-k)$ with coefficients $c \in \ell^{\infty}(\mathbb{Z})$ and let $N(f)=\{x \in \mathbb{R}:$ $f(x)=0\}$ be the zero set of $f$. Then either $D^{-}(N(f)) \leq 1$ or $f \equiv 0$. 
This statement resembles the classical result on the density of zeros of an entire function of exponential type $[13,46]$, but its proof is surprisingly indirect and makes little use of complex analysis methods. Theorem 1.6 can also be seen in the context of the variation-diminishing property of totally positive functions $[52,53]$.

\subsection{Methods}

We draw on methods from different areas and exploit new interplays between them.

(i) Structure theory of Gabor frames. The duality theory of Gabor frames [51] in the formulation of Janssen [35] relates the frame property of $\mathcal{G}(g, \alpha \mathbb{Z} \times \beta \mathbb{Z})$ to a (uniform) sampling problem in the shift-invariant space $V^{2}(g)$ (with shifts by $\beta^{-1} \mathbb{Z}$ instead of $\mathbb{Z}$ ). We extend this connection to nonuniform sampling sets and derive a characterization of semi-regular Gabor frames, which we formulate in a coarse version as follows.

Lemma $\mathcal{G}(g,(-\Lambda) \times \mathbb{Z})$ is a frame for $L^{2}(\mathbb{R})$, if and only if $x+\Lambda$ is a sampling set for $V^{2}(g)$ for all $x$ with uniform constants.

In a crucial step, we will relate a uniqueness problem of the form " $f \equiv 0$ on $\Lambda \Rightarrow f \equiv 0$ on $\mathbb{R}$ " in a shift-invariant space to the completeness of a Gabor system in a suitable function space (Theorem 2.6).

(ii) Frames and sampling without inequalities. We will derive several new abstract characterizations for sampling sets in shift-invariant spaces that do not require an inequality (Theorem 3.1). These characterizations are inspired by Beurling's approach to bandlimited functions and deal with the subtle interplay between sampling on the Hilbert space $V^{2}(g)$ and uniqueness on a larger space associated with the $L^{\infty}$-norm. While in the Paley-Wiener space there is an explicit bandwidth parameter that permits such arguments, no direct ana$\log$ is available for shift-invariant spaces. To circumvent this obstruction, we use a non-commutative version of Wiener's Lemma and the spectral invariance of matrices with $\ell^{1}$-off-diagonal decay. The depth of the characterization of sampling sets without inequality is hidden in these results about spectral invariance, which go back to Baskakov [7] and Sjöstrand [58].

(iii) Totally positive functions. We will use Schoenberg's characterization of totally positive functions by their Fourier-Laplace transform [52], but not the total positivity itself.

It is worthwhile to compare the methods and results for totally positive functions of finite type in [29] (where $\left.\hat{g}(\xi)=\prod_{j=1}^{n}\left(1+2 \pi i \delta_{j} \xi\right)^{-1}\right)$ to those for totally positive functions of Gaussian type. For totally positive functions of finite type we made essential use of the total positivity and the Schoenberg-Whitney conditions to reduce the Gabor frame property to a finite- 
dimensional problem. Corollary 1.5 remains true, whereas the statements of Theorems 1.2, 1.4 and 1.6 as stated are false for finite type.

Therefore we developed a completely new proof strategy for our results on totally positive functions of Gaussian type. The line of arguments is quite nonlinear and switches between sampling in shift-invariant spaces and Gabor frames. We first prove Theorem 1.2 for the special case of a Gaussian generator $\varphi(x)=e^{-c x^{2}}$. For this we will invoke the fundamental characterization of sampling sets in Bargmann-Fock space by Lyubarskii and Seip [47,56], and in fact, slightly elaborate on their main argument that relates density of zeros and growth. We then show that $V^{2}(g)$ for totally positive $g$ of Gaussian type is mapped onto $V^{2}(\varphi)$ by a suitable differential operator. This fact allows us to compare the density of the zero sets of functions in the corresponding shiftinvariant spaces. The general version of Theorem 1.2 then follows from the characterization of sampling sets without inequalities. Finally we translate the sampling results for the shift-invariant spaces $V^{2}(g)$ into the characterization of Gabor frames of Theorem 1.4.

The paper is organized as follows. Section 2 introduces shift-invariant spaces and the connections to Gabor analysis. Section 3 develops a characterization of sampling sets without inequalities in the spirit of Beurling. These tools are exploited in Sect. 4 to obtain sampling and interpolation results in shift-invariant spaces with a Gaussian generator and some necessary technical extensions. The density of zero sets for functions in shift-invariant spaces with different generators is compared in Sect. 5, leading to the main results on sampling (Theorem 1.2). Finally, Sect. 6 contains the characterization of semi-regular Gabor frames (Theorem 1.4). In the Appendix we supply the postponed proofs.

\section{Sampling in shift-invariant spaces and nonuniform Gabor families}

We first set up the basics about shift-invariant spaces, formulate several structural characterizations and point out some connections between shift-invariant spaces and Gabor families. For future reference we remark that the results in this section hold equally for sampling in shift-invariant spaces in higher dimensions (with identical proofs).

\subsection{Shift-invariant spaces}

Let $g: \mathbb{R} \rightarrow \mathbb{C}$ be a function in the Wiener amalgam space $W_{0}=W\left(C, \ell_{1}\right)$, i.e., $g$ is continuous and

$$
\|g\|_{W}:=\sum_{k \in \mathbb{Z}} \max _{x \in[k, k+1]}|g(x)|<\infty .
$$


In the center of our study are the shift-invariant spaces

$$
V^{p}(g)=\left\{\sum_{k \in \mathbb{Z}} c_{k} g(\cdot-k):\left(c_{k}\right)_{k \in \mathbb{Z}} \in \ell^{p}(\mathbb{Z})\right\}
$$

for $1 \leq p \leq \infty$. A comprehensive survey on shift-invariant spaces was given by Ron in [50].

Standard computations using Hölder's inequality [3] show that

$$
\left\|\sum_{k \in \mathbb{Z}} c_{k} g(\cdot-k)\right\|_{p} \leq\|g\|_{W}\|c\|_{p}, \quad c \in \ell^{p}(\mathbb{Z}),
$$

and consequently $V^{p}(g) \subseteq L^{p}(\mathbb{R})$.

Throughout we will assume and use the following stability of the generator $g$.

Theorem 2.1 Let $g \in W_{0}$. Then the following are equivalent.

(a) There exists $p \in[1, \infty]$ and $C_{p}>0$ such that

$$
\left\|\sum_{k \in \mathbb{Z}} c_{k} g(\cdot-k)\right\|_{p} \geq C_{p}\|c\|_{p}, \quad c \in \ell^{p}(\mathbb{Z}) .
$$

(b) For all $p \in[1, \infty]$ there exists a constant $C_{p}>0$ such that

$$
\left\|\sum_{k \in \mathbb{Z}} c_{k} g(\cdot-k)\right\|_{p} \geq C_{p}\|c\|_{p}, \quad c \in \ell^{p}(\mathbb{Z}) .
$$

(c) The shifts $\{g(\cdot-k): k \in \mathbb{Z}\}$ are $\ell^{\infty}$-independent; this means that $\sum_{k \in \mathbb{Z}} c_{k} g(\cdot-k) \neq 0$ for every $c \in \ell^{\infty}(\mathbb{Z}) \backslash\{0\}$.

(d) For every $\xi \in \mathbb{R}$ we have $\sum_{k \in \mathbb{Z}}|\hat{g}(\xi+k)|^{2}>0$.

We say that $g$ has stable integer shifts if any of the equivalent conditions of Theorem 2.1 holds. The equivalence was proved in [39, Theorem 3.5] and [50, Theorem 29] under a slightly more general condition on $g$.

\subsection{Sampling, uniqueness, and interpolating sets for shift-invariant spaces}

Since for $g \in W_{0}$ all spaces $V^{p}(g)$ are subspaces of $C(\mathbb{R})$, sampling of functions $f \in V^{p}(g)$ is well-defined. A subset $\Lambda \subseteq \mathbb{R}$ is called relatively separated, if

$$
n_{\Lambda}:=\max _{x \in \mathbb{R}} \#(\Lambda \cap[x, x+1])<\infty,
$$


and it is called separated if $\inf \{|\lambda-\mu|: \lambda, \mu \in \Lambda, \lambda \neq \mu\}=\delta>0$. If follows that every relatively separated set can be partitioned into $n_{\Lambda}$ separated subsets $\Lambda_{j}, j=1, \ldots, n_{\Lambda}$, with separation constant $1 / 2$.

Clearly, for a relatively separated set $\Lambda$ the lower Beurling density

$$
D^{-}(\Lambda):=\liminf _{R \rightarrow \infty} \inf _{x \in \mathbb{R}} \frac{\#(\Lambda \cap[x-R, x+R])}{2 R}
$$

and the upper Beurling density $D^{+}(\Lambda)$ (with lim sup and sup in its definition) are finite.

Let $g \in W_{0}$ have stable integer shifts. A relatively separated set $\Lambda \subseteq \mathbb{R}$ is called a sampling set for the shift-invariant space $V^{p}(g), 1 \leq p<\infty$, if there are constants $A_{p}, B_{p}>0$ such that

$$
A_{p}\|f\|_{p}^{p} \leq \sum_{\lambda \in \Lambda}|f(\lambda)|^{p} \leq B_{p}\|f\|_{p}^{p}
$$

for all $f \in V^{p}(g)$. For $p=\infty$, we require that

$$
A_{\infty}\|f\|_{\infty} \leq \sup _{\lambda \in \Lambda}|f(\lambda)| \leq B_{\infty}\|f\|_{\infty}
$$

holds for all $f \in V^{\infty}(g)$.

A set $\Lambda$ is called a uniqueness set for $V^{p}(g)$, if $f \in V^{p}(g)$ and $f(\lambda)=0$ for all $\lambda \in \Lambda$ implies that $f=0$.

If for every $a \in \ell^{p}(\Lambda)$ there exists an $f \in V^{p}(g)$ such that $f(\lambda)=a_{\lambda}$, then $\Lambda$ is said to be an interpolating set.

These notions can be expressed in terms of the matrices (called the preGramian in [51])

$$
P_{\Lambda}(g):=(g(\lambda-k))_{\lambda \in \Lambda, k \in \mathbb{Z}} .
$$

If $g \in W_{0}$, then by Schur's test, the matrix $P_{\Lambda}(g)$ defines a bounded linear operator $P_{\Lambda}(g): \ell^{p}(\mathbb{Z}) \rightarrow \ell^{p}(\Lambda), 1 \leq p \leq \infty$, and,

$$
\left\|P_{\Lambda}(g) c\right\|_{p} \leq n_{\Lambda}\|g\|_{W}\|c\|_{p}, \quad c \in \ell^{p}(\mathbb{Z})
$$

see for example [3]. The equivalence of norms $\left\|\sum_{k} c_{k} g(\cdot-k)\right\|_{p} \asymp\|c\|_{p}$ and the definitions imply the following characterizations.

Lemma 2.2 Assume that $g \in W_{0}$ has stable integer shifts. Let $1 \leq p \leq \infty$ and $\Lambda \subseteq \mathbb{R}$ be relatively separated. Then: 
(a) $\Lambda$ is a sampling set for $V^{p}(g)$, if and only if $P_{\Lambda}(g)$ is p-bounded below, i.e., if and only if there exist $\tilde{A}_{p}>0$ such that

$$
\left\|P_{\Lambda}(g) c\right\|_{p} \geq \tilde{A}_{p}\|c\|_{p} \text { for all } c \in \ell^{p}(\mathbb{Z})
$$

(b) $\Lambda$ is a uniqueness set for $V^{p}(g)$, if and only if $P_{\Lambda}(g)$ is one-to-one on $\ell^{p}(\mathbb{Z})$.

(c) $\Lambda$ is an interpolating set for $V^{p}(g)$, if and only if $P_{\Lambda}(g)$ is surjective from $\ell^{p}(\mathbb{Z})$ onto $\ell^{p}(\Lambda)$.

\subsection{The connection between sampling and Gabor analysis}

Sampling and interpolation problems in the shift-invariant space $V^{2}(g)$ are closely related to the construction of Gabor frames and Riesz bases. The connection is implicit in the duality theory by Janssen [35] and Ron and Shen [51] and has been instrumental in the characterization of Gabor frames on lattices for totally positive functions of finite type [29]. The main objective of this section is to extend the relation between Gabor analysis and shift-invariant space to (i) more nonuniform phase-space nodes and to (ii) uniqueness / completeness problems in appropriate function spaces.

We use $T_{y} g(x)=g(x-y)$ for translation and $M_{\xi} g(x)=e^{2 \pi i x \xi} g(x)$ for modulation. A Gabor family is a collection of time-frequency shifts, and we are particularly interested in the semi-regular family

$$
\mathcal{G}(g,(-\Lambda) \times \mathbb{Z})=\left\{M_{k} T_{-\lambda} g: k \in \mathbb{Z}, \lambda \in \Lambda\right\},
$$

associated with the window function $g$ and a set $\Lambda \subseteq \mathbb{R}$. By definition, this family is a Gabor frame, if there exist constants $A, B>0$, such that

$$
A\|f\|_{2}^{2} \leq \sum_{k \in \mathbb{Z}} \sum_{\lambda \in \Lambda}\left|\left\langle f, M_{k} T_{-\lambda} g\right\rangle\right|^{2} \leq B\|f\|_{2}^{2} \quad \text { for all } f \in L^{2}(\mathbb{R}) .
$$

We next formulate several characterizations of Gabor frames that establish the connection to sampling in shift-invariant spaces. We recall the definition of the Zak transform $Z g(x, \xi)=\sum_{k \in \mathbb{Z}} g(x+k) e^{2 \pi i k \xi}$ of a function $g \in L^{1}(\mathbb{R})$.

Theorem 2.3 Assume that $g \in W_{0}$ has stable integer shifts and that $\Lambda \subseteq \mathbb{R}$ is relatively separated. The following are equivalent.

(a) The family $\mathcal{G}(g,(-\Lambda) \times \mathbb{Z})$ is a frame for $L^{2}(\mathbb{R})$. 
(b) The set $\Lambda+x$ is a sampling set of $V^{2}(g)$ for every $x \in[0,1)$, i.e., for each $x \in[0,1)$, there exist $A_{x}, B_{x}>0$ such that

$$
A_{x}\|c\|_{2}^{2} \leq \sum_{\lambda \in \Lambda}\left|\sum_{k \in \mathbb{Z}} c_{k} g(\lambda+x-k)\right|^{2} \leq B_{x}\|c\|_{2}^{2} \text { for all } c \in \ell^{2}(\mathbb{Z}) .
$$

(c) There exist $A, B>0$ such that, for all $x \in[0,1)$,

$$
A\|c\|_{2}^{2} \leq \sum_{\lambda \in \Lambda}\left|\sum_{k \in \mathbb{Z}} c_{k} g(\lambda+x-k)\right|^{2} \leq B\|c\|_{2}^{2} \text { for all } c \in \ell^{2}(\mathbb{Z}) .
$$

(d) There exist constants $A, B>0$ such that

$$
A\|h\|_{2}^{2} \leq \sum_{\lambda \in \Lambda}\left|\int_{0}^{1} h(\xi) Z g(x+\lambda, \xi) d \xi\right|^{2} \leq B\|h\|_{2}^{2}
$$

for all $h \in L^{2}([0,1])$ and $x \in[0,1)$.

Moreover, the optimal constants in (2.3), (2.4), and (2.5) coincide.

The proof is postponed to the Appendix.

Remark 2.4 (i) For the case $\Lambda=\alpha \mathbb{Z}$, the equivalence of (a), (c), and (d) is Janssen's version of the duality theory [35]. It is one of the most useful tools for deriving concrete and strong results about Gabor frames [29,36]. The extension of the equivalences to nonuniform sampling sets is new and is derived by a suitable modification of the known proofs.

(ii) Condition (d) can be reformulated by saying that for every $x \in[0,1)$ the set of functions $\{Z g(x+\lambda, \xi): \lambda \in \Lambda\}$ is a frame for $L^{2}([0,1], d \xi)$.

Interpolation problems in shift-invariant spaces can similarly be related to Gabor systems. We state the following theorem, whose proof is given in the Appendix, together with the one of Theorem 2.3.

Theorem 2.5 Let $g \in W_{0}$ have stable integer shifts and $\Lambda \subseteq \mathbb{R}$ be relatively separated. Then the following are equivalent.

(a) $\mathcal{G}(g,(-\Lambda) \times \mathbb{Z})$ is a Riesz sequence in $L^{2}(\mathbb{R})$.

(b) For each $x \in[0,1), \Lambda+x$ is an interpolating set for $V^{2}(g)$.

(c) The sets $\Lambda+x, x \in[0,1)$, are interpolating sets for $V^{2}(g)$ with uniform constants, i.e., for all $a \in \ell^{2}(\Lambda)$ there exists $f_{x} \in V^{2}(g)$ such that $f_{x}(\lambda+x)=a_{\lambda}$, for all $\lambda \in \Lambda$, and $\left\|f_{x}\right\|_{2} \leq C\|a\|_{2}$, with a constant $C$ independent of $x$. 
The following theorem relates Gabor systems and shift-invariant spaces, but this time it concerns $L^{\infty}$-uniqueness. It serves as one of our main tools in the following sections.

Theorem 2.6 Let $g \in W_{0}(\mathbb{R})$ and let $\Lambda \subseteq \mathbb{R}$ be arbitrary. Suppose that $\mathcal{G}(g,(-\Lambda) \times \mathbb{Z})$ spans a dense subspace in $W_{0}(\mathbb{R})$. Then for every $x \in \mathbb{R}$, $\Lambda+x$ is a uniqueness set for $V^{\infty}(g)$.

Proof Suppose that $\operatorname{span} \mathcal{G}(g,(-\Lambda) \times \mathbb{Z})$ is dense in $W_{0}(\mathbb{R})$. For simplicity, we assume that $x=0$ (otherwise, we shift $\Lambda$ suitably). We need to show that the matrix $P_{\Lambda}(g)$ is one-to-one from $\ell^{\infty}(\mathbb{Z})$ to $\ell^{\infty}(\Lambda)$. By duality, this is equivalent to saying that the matrix $P_{\Lambda}(g)^{\prime}=(g(\lambda-k))_{k \in \mathbb{Z}, \lambda \in \Lambda}$ viewed as an operator from $\ell^{1}(\Lambda) \rightarrow \ell^{1}(\mathbb{Z})$ has dense range in $\ell^{1}(\mathbb{Z})$. Thus we need to show that for every $c \in \ell^{1}(\mathbb{Z})$ and $\epsilon>0$ there is a sequence $a \in \ell^{1}(\Lambda)$ such that $\left\|P_{\Lambda}(g)^{\prime} a-c\right\|_{1}<\epsilon$. Let $\eta \in C^{\infty}(\mathbb{R})$ be supported on $[-1 / 4,1 / 4]$ and $\eta(x)=1$ in a neighborhood of the origin, and let

$$
h=\sum_{k \in \mathbb{Z}} c_{k} \eta(\cdot+k)
$$

Then $h \in W_{0}$ and $h(-k)=c_{k}$ for all $k \in \mathbb{Z}$.

Given $\epsilon>0$, by assumption, there exists a finite linear combination

$$
f(x):=\sum_{\lambda \in \Lambda} \sum_{j \in \mathbb{Z}} b_{\lambda, j} e^{2 \pi i j x} g(x+\lambda)
$$

with coefficients $b \in \ell^{1}(\Lambda \times \mathbb{Z})$ (with finite support), such that $\|f-h\|_{W_{0}}<\epsilon$.

Let $a_{\lambda}:=\sum_{j} b_{\lambda, j}$, and note that $\|a\|_{\ell^{1}(\Lambda)} \leq\|b\|_{\ell^{1}(\Lambda \times \mathbb{Z})}<\infty$. In addition, $f(-k)=\sum_{\lambda \in \Lambda} \sum_{j \in \mathbb{Z}} b_{\lambda, j} g(\lambda-k)=\left(P_{\Lambda}(g)^{\prime} a\right)_{k}$. Finally, we estimate

$$
\left\|c-P_{\Lambda}(g)^{\prime} a\right\|_{\ell^{1}(\mathbb{Z})}=\left\|\left.(f-h)\right|_{\mathbb{Z}}\right\|_{\ell^{1}(\mathbb{Z})} \leq\|f-h\|_{W_{0}}<\varepsilon .
$$

Hence the desired approximation holds.

\section{Characterization of Beurling type for sampling sets and Gabor frames}

\subsection{Characterization of sampling sets for shift-invariant spaces}

According to (2.1), a sampling set for $V^{p}(g)$ is also a uniqueness set for $V^{p}(g)$. The sampling inequalities in (2.1) are stronger than mere uniqueness: they quantify the stability of the map $f \mid \Lambda \mapsto f$. It is a remarkable insight due to Beurling [10], [11, p. 351-365] that sampling sets for bandlimited functions can be characterized in terms of uniqueness sets for a larger space of functions. 
We now develop an instance of this principle in the context of shift-invariant spaces and relate a sampling problem on $V^{2}(g)$ to a collection of uniqueness problems on $V^{\infty}(g)$. Analogous results hold for Gabor frames [25,28].

For the formulation, we recall Beurling's notion of a weak limit of a sequence of sets. A sequence $\left\{\Lambda_{n}: n \geq 1\right\}$ of subsets of $\mathbb{R}$ is said to converge weakly to a set $\Lambda \subseteq \mathbb{R}$, denoted $\Lambda_{n} \stackrel{w}{\rightarrow} \Lambda$, if for every open bounded interval $(a, b)$ and every $\varepsilon>0$, there exist $n_{0} \in \mathbb{N}$ such that for all $n \geq n_{0}$.

$$
\Lambda_{n} \cap(a, b) \subseteq \Lambda+(-\varepsilon, \varepsilon) \text { and } \Lambda \cap(a, b) \subseteq \Lambda_{n}+(-\varepsilon, \varepsilon) .
$$

The notion of weak convergence of sets is closely related to local convergence in the Hausdorff metric, but the precise relation is slightly technical (see [28, Section 4].

Given a set $\Lambda \subseteq \mathbb{R}$, we let $W_{\mathbb{Z}}(\Lambda)$ denote the collection of all weak limits of integer translates of $\Lambda$, i.e., $\Gamma \in W_{\mathbb{Z}}(\Lambda)$, if there exists a sequence $\left\{k_{n}\right.$ : $n \geq 1\} \subseteq \mathbb{Z}$ such that $\Lambda+k_{n} \stackrel{w}{\rightarrow} \Gamma$. If $\Lambda$ is relatively separated, each set in $W_{\mathbb{Z}}(\Lambda)$ is also relatively separated (see, e.g., [28, Lemma 4.5]).

The following characterization of sampling sets for $V^{p}(g)$ is new and should be compared with Theorem 2.1.

Theorem 3.1 Let $g \in W_{0}$ have stable integer shifts and let $\Lambda \subseteq \mathbb{R}$ be a relatively separated set. The following are equivalent:

(a) $\Lambda$ is a sampling set of $V^{p}(g)$ for some $p \in[1, \infty]$.

(b) $\Lambda$ is a sampling set of $V^{p}(g)$ for all $p \in[1, \infty]$.

(c) Every $\Gamma \in W_{\mathbb{Z}}(\Lambda)$ is a sampling set for $V^{\infty}(g)$.

(d) Every $\Gamma \in W_{\mathbb{Z}}(\Lambda)$ is a uniqueness set for $V^{\infty}(g)$.

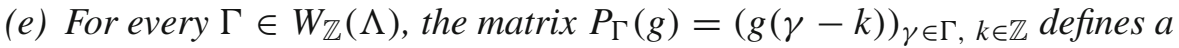
bounded injective operator from $\ell^{\infty}(\mathbb{Z})$ to $\ell^{\infty}(\Gamma)$.

(f) For every $\Gamma \in W_{\mathbb{Z}}(\Lambda)$, the matrix $P_{\Gamma}(g)^{\prime}=(g(\gamma-k))_{k \in \mathbb{Z}, \gamma \in \Gamma \text { defines }}$ an operator $\ell^{1}(\Gamma) \rightarrow \ell^{1}(\mathbb{Z})$ with dense range.

The Proof of Theorem 3.1 relies on the theory of localized frames [24] and combines facts about spectral invariance with manipulations with weak limits. As we have proved a similar characterization without inequalities for Gabor frames in [28, Section 5], we postpone the proof to the Appendix.

Remark 3.2 (i) Theorem 3.1 says that instead of proving an inequality of type (1.1) or the left-invertibility of the matrix $P_{\Lambda}(g)$, it suffices to verify that $P_{\Gamma}(g)$ has trivial kernel on the larger space $\ell^{\infty}(\mathbb{Z})$ for every $\Gamma \in W_{\mathbb{Z}}(\Lambda)$. This is conceptually easier.

(ii) The $\ell^{\infty}$-injectivity in (d) cannot be replaced by $\ell^{2}$-injectivity. This is in accordance with Theorem 2.1 where $\ell^{p}$-injectivity of $c \mapsto$ $\sum_{k \in \mathbb{Z}} c_{k} g(\cdot-k)$ is a much weaker condition for $p<\infty$. Also note 
that the equivalence can hold only for $g \in W_{0}$, otherwise some of the conditions do not even make sense.

(iii) We emphasize that Theorem 3.1 does not say that every uniqueness set for $V^{\infty}(g)$ is a sampling set. See Remark 4.2 below for a concrete example.

(iv) The exact analog of Theorem 3.1 is not valid for the Paley-Wiener space, because the sampling problems associated with different $L^{p}$-norms are not equivalent. In our case, the assumption $g \in W_{0}$ allows us to invoke a matrix variant of Wiener's $1 / f$ lemma due to Sjöstrand [58].

\subsection{Characterization of Gabor frames}

Although not needed for the main results, we formulate a new characterization of Gabor frames. In the formulation we again need weak limits of sequences of sets. For a set $\Lambda \subseteq \mathbb{R}$, we define $W(\Lambda)$ as the set of weak limits of translates $\Lambda+$ $x$, i.e., $\Gamma \in W(\Lambda)$, if and only if there exists a sequence of real numbers $\left\{x_{n}\right.$ : $n \geq 1\} \subseteq \mathbb{R}$, such that $\Lambda+x_{n} \stackrel{w}{\rightarrow} \Gamma$. We note that $W(\Lambda)=\bigcup_{x \in[0,1]} W_{\mathbb{Z}}(\Lambda+$ $x)$.

Theorem 3.3 Assume that $g \in W_{0}$ has stable integer shifts and that $\Lambda \subseteq \mathbb{R}$ is relatively separated. The following are equivalent.

(a) The family $\mathcal{G}(g,(-\Lambda) \times \mathbb{Z})$ is a frame for $L^{2}(\mathbb{R})$.

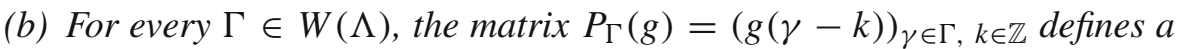
bounded injective operator from $\ell^{\infty}(\mathbb{Z})$ to $\ell^{\infty}(\Gamma)$.

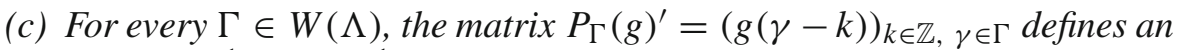
operator $\ell^{1}(\Gamma) \rightarrow \ell^{1}(\mathbb{Z})$ with dense range.

Theorem 3.3 is a direct consequence of Theorems 2.3 and 3.1.

\section{Nonuniform sampling and Gabor families with Gaussian generators}

Let $\varphi(x)=e^{-c x^{2}}, c>0$, be a scaled centered Gaussian. Lyubarskii [47] and Seip [56] proved the following.

Theorem 4.1 (Lyubarskii, Seip) Let $\Omega \subseteq \mathbb{R}^{2}$ be separated. Then

(a) $\mathcal{G}(\varphi, \Omega):=\left\{M_{\xi_{2}} T_{\xi_{1}} \varphi:\left(\xi_{1}, \xi_{2}\right) \in \Omega\right\}$ is a frame for $L^{2}(\mathbb{R})$ if and only if

$$
D_{2}^{-}(\Omega):=\liminf _{R \rightarrow \infty} \inf _{z \in \mathbb{R}^{2}} \frac{\#\left(\Omega \cap\left(z+[-R, R]^{2}\right)\right)}{4 R^{2}}>1,
$$

and 
(b) $\mathcal{G}(\varphi, \Omega)$ is a Riesz sequence if and only if

$$
D_{2}^{+}(\Omega):=\limsup _{R \rightarrow \infty} \sup _{z \in \mathbb{R}^{2}} \frac{\#\left(\Omega \cap\left(z+[-R, R]^{2}\right)\right)}{4 R^{2}}<1 .
$$

Remark 4.2 While a set $\Omega$ generating a Gabor Riesz sequence is necessarily separated, a set generating a frame may be only relatively separated, and Theorem 4.1 (a) does not directly apply to non-separated sets. The exact characterization is the following: $\mathcal{G}(\varphi, \Omega)$ is a frame of $L^{2}(\mathbb{R})$ if and only if $\Omega$ is relatively separated and contains a separated subset of density $>1$.

To see the relevance of this subtle distinction, we consider the set $\Omega=$ $\left\{(k, j),\left(k+2^{-|k|}, j\right): k, j \in \mathbb{Z}\right\}$. Then clearly $\Omega$ is relatively separated and $D^{-}(\Omega)=2$, but $\mathcal{G}(\varphi, \Omega)$ is not a frame of $L^{2}(\mathbb{R})$. Indeed, if $\mathcal{G}(\varphi, \Omega)$ were a frame of $L^{2}(\mathbb{R})$, then by [28, Theorem 5.1] (equivalence of (i) and (v)) $\mathcal{G}\left(\varphi, \Omega^{\prime}\right)$ would be a frame for every $\Omega^{\prime} \in W(\Omega)$. However, it is easy to see that $\mathbb{Z}^{2} \in W(\Omega)$ and it is well-known that $\mathcal{G}\left(\varphi, \mathbb{Z}^{2}\right)$ is not a frame $[9,32]$.

In spite of the previous remark, we have the following result.

Theorem 4.3 Let $\Omega \subseteq \mathbb{R}^{2}$ be an arbitrary set with $D^{-}(\Omega)>1$. Then $\mathcal{G}(\varphi, \Omega)$ spans a dense subspace of $W_{0}(\mathbb{R})$.

The Proof of Theorem 4.3 uses (i) the connection between Gabor systems with a Gaussian generator and sampling in the Bargmann-Fock space of entire functions, provided by the Bargmann transform [6,16], and (ii) a complex analysis argument that compares growth and density of zeros that goes back to Beurling [10,11], and in the form required here was given by Brekke and Seip [14]. Since the claim that the result is valid for possibly non-separated sets is an essential technical point not explicitly stated in the references, we sketch a self-contained argument in the appendix.

We have now assembled all tools to prove a general result for the shiftinvariant spaces $V^{p}(\varphi)$ in the style of Beurling. As a consequence of Theorem 2.3, we obtain the following statement, which implies Theorem 1.3 (announced in the Introduction).

Theorem 4.4 Let $\varphi(x)=e^{-c x^{2}}$ with $c>0$.

(a) If $\Lambda \subseteq \mathbb{R}$ has density $D^{-}(\Lambda)>1$, then $\Lambda$ is a uniqueness set for $V^{\infty}(\varphi)$.

(b) If $\Lambda$ is separated and $D^{-}(\Lambda)>1$, then $\Lambda$ is a sampling set for $V^{p}(\varphi)$ for $1 \leq p \leq \infty$.

(c) If $\Lambda$ is separated and $D^{+}(\Lambda)<1$, then $\Lambda$ is an interpolating set for $V^{2}(\varphi)$.

Proof (a) Assume that $D^{-}(\Lambda)>1$. Then the set $\Omega=(-\Lambda) \times \mathbb{Z} \subseteq \mathbb{R}^{2}$ has lower Beurling density 


$$
D_{2}^{-}((-\Lambda) \times \mathbb{Z})=D^{-}(-\Lambda)=D^{-}(\Lambda)>1
$$

(where as before $D_{2}^{-}$is the Beurling density of a subset in $\mathbb{R}^{2}$, and $D^{-}$is the Beurling density of a subset in $\mathbb{R})$.

By Theorem 4.3, $\mathcal{G}(\varphi,(-\Lambda) \times \mathbb{Z})$ spans a dense subspace of $W_{0}(\mathbb{R})$. Theorem 2.6 now implies that $x+\Lambda$ is a uniqueness set for $V^{\infty}(\varphi)$ for all $x \in \mathbb{R}$.

(b) If $D^{-}(\Lambda)>1$ and, in addition, $\Lambda$ is separated, then, by Theorem 4.1, $\mathcal{G}(\varphi,(-\Lambda) \times \mathbb{Z})$ is a frame for $L^{2}(\mathbb{R})$. Hence $\Lambda$ is a sampling set for $V^{2}(\varphi)$ by Theorem 2.3 .

Part (c) follows similarly, this time we invoke Theorem 2.5.

Remark 4.5 (i) The functions in $V^{2}(\varphi)$ and $V^{\infty}(\varphi)$ are certain entire functions of order 2. It would be interesting to find a direct proof without the detour to Gabor frames in Theorem 4.3.

(ii) The critical case $\Lambda=x+\mathbb{Z}$ (hence $D^{-}(\Lambda)=1$ ) for arbitrary generator $g \in W_{0}$ is easy to understand with characterization (d) of Theorem 2.3. The quasi-periodicity of the Zak transform implies that $Z g(x+k, \xi)=$ $e^{-2 \pi i k \xi} Z g(x, \xi)$. It follows that $x+\Lambda$ is a sampling set, if and only if the set $\left\{e^{2 \pi i k \xi} Z g(x, \xi): k \in \mathbb{Z}\right\}$ is a frame for $L^{2}(\mathbb{T}, d \xi)$. This is the case, if and only if $Z g(x, \xi) \neq 0$ for all $\xi \in \mathbb{R}$. However, since $g \in W_{0}$ has a continuous Zak transform, there exists at least one point $\left(x_{0}, \xi_{0}\right)$ such that $Z g\left(x_{0}, \xi_{0}\right)=0$ (see e.g. [9]). Consequently, $x_{0}+\Lambda$ is not a sampling set. This case was investigated and understood early on by Janssen [33], Walter [61], and Baxter and Sivakumar [8].

\section{Nonuniform sampling with totally positive generator}

Next we study the sampling problem in shift-invariant spaces with a totally positive function of Gaussian type as a generator. As explained in the introduction, these are all (totally positive) functions $g$ whose Fourier transform factors as

$$
\hat{g}(\xi)=\prod_{j=1}^{n}\left(1+2 \pi i \delta_{j} \xi\right)^{-1} e^{-c \xi^{2}}, \quad \delta_{1}, \ldots, \delta_{n} \in \mathbb{R}, c>0 .
$$

By Schoenberg's characterization [52], every $g$ of this form is totally positive, and every totally positive function in $L^{1}(\mathbb{R})$ is a limit of totally positive functions of Gaussian type. In addition, we note that $g$ has stable integer shifts. This can be easily verified using condition (d) of Theorem 2.1 and the fact that the Fourier transform of $g$ in (5.1) has no real zeros. 
Next, we denote the zero set of a continuous function $f$ by

$$
N_{f}:=\{x \in \mathbb{R}: f(x)=0\} .
$$

The identity operator is denoted by $I$ as usual.

The following lemma is the crucial insight that allows us to go from the Gaussian to totally positive functions of Gaussian type as generators.

Lemma 5.1 Let $f \in C^{1}(\mathbb{R})$ be real-valued. For $a \in \mathbb{R}, a \neq 0$, let $g=$ $\left(I+a \frac{d}{d x}\right) f$. Then

$$
D^{-}\left(N_{g}\right) \geq D^{-}\left(N_{f}\right)
$$

Proof Note that $I+a \frac{d}{d x}=a e^{-x / a} \frac{d}{d x} e^{x / a}$. We define $h \in C^{1}(\mathbb{R})$ by $h(x)=$ $a e^{x / a} f(x)$ and note that $N_{h}=N_{f}$. Furthermore, since

$$
g(x)=\left(I+a \frac{d}{d x}\right) f(x)=e^{-x / a} h^{\prime}(x),
$$

we conclude that $N_{g}=N_{h^{\prime}}$. It remains to show that $D^{-}\left(N_{h^{\prime}}\right) \geq D^{-}\left(N_{h}\right)$.

Let $x \in \mathbb{R}, R>0$, and $F \subseteq N_{h} \cap[x-R, x+R]$ a finite subset. By Rolle's theorem,

$$
\#\left(N_{h^{\prime}} \cap[x-R, x+R]\right) \geq \# F-1 .
$$

Since this holds for every finite subset $F \subseteq N_{h} \cap[x-R, x+R]$, it follows that $\#\left(N_{h^{\prime}} \cap[x-R, x+R]\right) \geq \#\left(N_{h} \cap[x-R, x+R]\right)-1$. Therefore, $D^{-}\left(N_{h^{\prime}}\right) \geq D^{-}\left(N_{h}\right)$, and

$$
D^{-}\left(N_{g}\right)=D^{-}\left(N_{h^{\prime}}\right) \geq D^{-}\left(N_{h}\right)=D^{-}\left(N_{f}\right),
$$

as claimed.

We now state our main results on sampling, which are an extended form of Theorems 1.2 and 1.6 in the introduction.

Theorem 5.2 Let $g$ be a totally positive function of Gaussian type.

(a) If $\Lambda \subseteq \mathbb{R}$ has density $D^{-}(\Lambda)>1$, then $\Lambda$ is a uniqueness set for $V^{\infty}(g)$.

(b) If $\Lambda$ is separated and $D^{-}(\Lambda)>1$, then $\Lambda$ is a sampling set for $V^{2}(g)$.

Proof Recall that $g$ is real-valued and has stable integers shifts.

(a) Let $c \in \ell^{\infty}(\mathbb{Z})$ and assume that $f=\sum_{k \in \mathbb{Z}} c_{k} g(\cdot-k) \in V^{\infty}(g)$ vanishes on $\Lambda$ with $D^{-}(\Lambda)>1$. We want to show that $f \equiv 0$. Note that $f \in$ $C^{\infty}(\mathbb{R})$. Since $g$ is real-valued, we may assume without loss of generality that $f$ is also real-valued (by replacing $c_{k}$ by $\Re\left(c_{k}\right)$ or $\Im\left(c_{k}\right)$ if necessary). 
The representation of $\hat{g}$ in (5.1) implies that

$$
\prod_{j=1}^{n}\left(I+\delta_{j} \frac{d}{d x}\right) g=\varphi,
$$

where $\widehat{\varphi}(\xi)=e^{-c \xi^{2}}$. Now set

$$
\Phi=\sum_{k \in \mathbb{Z}} c_{k} \varphi(\cdot-k)
$$

Since $\varphi, g$ and their derivatives decay exponentially, we may interchange summation and differentiation in $f$, and obtain that

$$
\Phi=\prod_{j=1}^{n}\left(I+\delta_{j} \frac{d}{d x}\right) f .
$$

We are now in a position to apply Lemma 5.1 repeatedly and conclude that

$$
D^{-}\left(N_{\Phi}\right) \geq D^{-}\left(N_{f}\right) \geq D^{-}(\Lambda)>1 .
$$

By Theorem 4.4, the set $N_{\Phi}$ is a uniqueness set for $V^{\infty}(\varphi)$. Since obviously $\Phi \equiv 0$ on $N_{\Phi}$, we conclude that $\Phi \equiv 0$. Hence $c=0$ and therefore $f \equiv 0$. Thus $\Lambda$ is a uniqueness set for $V^{\infty}(g)$.

(b) Now assume that $\Lambda$ is separated. By Theorem 3.1, in order to show that $\Lambda$ is a sampling set for $V^{2}(g)$, it suffices to show that every $\Gamma \in W_{\mathbb{Z}}(\Lambda)$ is a uniqueness set for $V^{\infty}(g)$. Let $\Gamma \in W_{\mathbb{Z}}(\Lambda)$. Since $\Lambda$ is separated, the weak-limit $\Gamma$ is also separated and it satisfies $D^{-}(\Gamma) \geq D^{-}(\Lambda)>1$ (by Lemma 7.1 in the appendix). Part (a) now asserts that $\Gamma$ is a uniqueness set for $V^{\infty}(g)$. Therefore, by Theorem 3.1, $\Lambda$ is a sampling set for $V^{2}(g)$.

Remark 5.3 In the above proof, we do not know if the set $N_{\Phi}$ is separated, even if the original set $\Lambda$ is. Therefore we cannot conclude directly that $N_{\Phi}$ is a sampling set for $V^{2}(\varphi)$, but only that $N_{\Phi}$ is a uniqueness set for $V^{\infty}(\varphi)$. In this way, the proof exploits the full strength of Theorems 3.1 and 4.3.

Using a clever trick by Janssen and Strohmer [38], we obtain the following generalization of Theorem 5.2. Let $g$ be a totally positive function of Gaussian type and let $c, d \in \ell^{1}(\mathbb{Z})$ be two sequences with Fourier series $\hat{c}(\xi)=\sum_{k \in \mathbb{Z}} c_{k} e^{2 \pi i k \xi}$ and $\hat{d}$, such that

$$
\inf _{\xi \in \mathbb{R}}|\hat{c}(\xi)|>0 \quad \text { and } \inf _{\xi \in \mathbb{R}}|\hat{d}(\xi)|>0 .
$$


Now set

$$
\gamma=\sum_{k, l \in \mathbb{Z}} c_{k} d_{l} T_{k} M_{l} g
$$

Corollary 5.4 Let $\gamma$ be defined as in (5.2) and $\Lambda \subseteq \mathbb{R}$ be a separated set with density $D^{-}(\Lambda)>1$. Then $\Lambda$ is a sampling set for $V^{2}(\gamma)$.

In particular, if $\gamma(x)=\operatorname{sech}(v x)=2\left(e^{\nu x}+e^{-v x}\right)^{-1}$ and $D^{-}(\Lambda)>1$, then $\Lambda$ is a sampling set for $V^{2}(\gamma)$.

Proof Since $c, d \in \ell^{1}(\mathbb{Z})$, it is easy to see that $\gamma \in W_{0}$. Therefore the characterization in Theorem 3.1 is applicable. From the quasi-periodicity of the Zak transform we obtain that

$$
Z \gamma(x, \xi)=\hat{c}(-\xi) \hat{d}(x) Z g(x, \xi) .
$$

Since $|\hat{c}|,|\hat{d}| \geq C>0$ and $Z g$ satisfies the condition (2.5) of Theorem 2.3, it follows that $Z \gamma$ also satisfies condition (2.5). Therefore $\Lambda$ is a sampling set for $V^{2}(\gamma)$.

Finally, Janssen and Strohmer [38] showed that the hyperbolic secant $\operatorname{sech}(v x)$ possesses a representation (5.2) with respect to the Gaussian for all $v>0$, whence the statement follows.

\section{Gabor frames with totally positive generator}

We now use the relation between sampling sets for shift-invariant spaces and Gabor frames to derive a new result about Gabor frames with totally positive windows.

Despite intensive research about Gabor frames, the only complete results cover the Gaussian $\varphi(x)=e^{-c x^{2}}$, the hyperbolic secant $g(x)=\left(e^{c x}+\right.$ $\left.e^{-c x}\right)^{-1}$, and totally positive functions of finite type, which are defined by their Fourier transform as $\hat{g}(\xi)=\prod_{j=1}^{n}\left(1+2 \pi i \delta_{j} \xi\right)^{-1}, \delta_{1}, \ldots, \delta_{n} \in \mathbb{R} \backslash\{0\}$. Our current knowledge can be summarized as follows:

Theorem 6.1 Let $g$ be a Gaussian, the hyperbolic secant, or a totally positive function of finite type $n \geq 2, n \in \mathbb{N}$. Then the Gabor family $\mathcal{G}(g, \alpha \mathbb{Z} \times \beta \mathbb{Z})$ is a frame for $L^{2}(\mathbb{R})$ if and only if $\alpha \beta<1$.

The result goes back to $[34,38,47,56]$, the case of totally positive functions of finite type was settled in [29] with a new proof in [41]. The case $n=1$ corresponds to the one-sided exponential function $h(x)=e^{-\delta x} \chi_{[0, \infty)}(\delta x)$ and was already settled by Janssen [36]: $\mathcal{G}(h, \alpha \mathbb{Z} \times \beta \mathbb{Z})$ is a frame, if and only if $\alpha \beta \leq 1$.

The method for the proof in [29] uses techniques from approximation theory, namely the Schoenberg-Whitney conditions in $[29,55]$ for the invertibility of 
certain submatrices of the pre-Gramian $P_{\Lambda}(g)$. Note that the lattice $\Omega=$ $\alpha \mathbb{Z} \times \beta \mathbb{Z}$ has Beurling density $D^{-}(\Omega)=(\alpha \beta)^{-1}$, so that $\alpha \beta<1$ is precisely the density condition $D^{-}(\Omega)>1$.

We extend Theorem 6.1 in two ways: we use totally positive functions of Gaussian type as window functions, and we study semi-regular sets of the form $\Lambda \times \beta \mathbb{Z}$ instead of lattices $\alpha \mathbb{Z} \times \beta \mathbb{Z}$. The following implies Theorem 1.4 of the introduction.

Theorem 6.2 Let $g$ be a totally positive function of Gaussian type and let $\Lambda \subseteq \mathbb{R}$ be a separated set. Then the Gabor family $\mathcal{G}(g, \Lambda \times \beta \mathbb{Z})$ is a frame for $L^{2}(\mathbb{R})$ if and only if $0<\beta<D^{-}(\Lambda)$.

Proof We use the scaling invariance of the class of totally positive functions of Gaussian type: if $g$ is of the form (5.1), then the function $g_{\beta}(x)=g(x / \beta)$ is of the same form (5.1) with different parameters and a positive normalization factor. By the usual dilation argument $\mathcal{G}(g, \Lambda \times \beta \mathbb{Z})$ is a frame if and only if $\mathcal{G}\left(g_{\beta}, \beta \Lambda \times \mathbb{Z}\right)$ is a frame. Moreover, simple geometric facts yield

$$
D_{2}^{-}(\beta \Lambda \times \mathbb{Z})=D_{2}^{-}(\Lambda \times \beta \mathbb{Z})=D^{-}(\beta \mathbb{Z}) D^{-}(\Lambda)=\frac{D^{-}(\Lambda)}{\beta} .
$$

The combination of Theorems 2.3 and 5.2 implies that the Gabor family $\mathcal{G}\left(g_{\beta}, \beta \Lambda \times \mathbb{Z}\right)$ is a frame for $L^{2}(\mathbb{R})$, whenever $0<\beta<D^{-}(\Lambda)$.

The necessity of the condition $D^{-}(\Lambda \times \beta \mathbb{Z})>1$ follows from recent nonuniform versions of the Balian-Low theorem [4,28], which are applicable because $g$ satisfies the decay and integrability condition

$$
\int_{\mathbb{R}^{2}}\left|\left\langle g, M_{\xi} T_{x} g\right\rangle\right| d x d \xi<\infty .
$$

(In the standard jargon of time-frequency analysis, this condition means that $g$ belongs to the modulation space $M^{1}(\mathbb{R})$.) This completes the proof.

\section{Appendix}

\subsection{Density, separation and weak convergence}

In the following we need the dual space $W_{0}^{*}$. We can identify the dual space of $W_{0}$ with the space of complex-valued Radon measures $\mu$ such that

$$
\sup _{x \in \mathbb{R}}\|\mu\|_{C^{*}([x, x+1])}=\sup _{x \in \mathbb{R}}|\mu|([x, x+1])<\infty .
$$

In the usual notation of amalgam spaces the dual is $W_{0}^{*}=W\left(\mathcal{M}, \ell^{\infty}\right)[19,31]$. For completeness, we prove the following folklore lemma. 
Lemma 7.1 Let $\Lambda \subseteq \mathbb{R}$ be separated and $\Gamma \in W(\Lambda)$. Then $\Gamma$ is separated and $D^{-}(\Gamma) \geq D^{-}(\Lambda)$.

Proof Let $\left\{x_{n}: n \geq 1\right\} \subseteq \mathbb{R}$ be such that $\Lambda+x_{n} \stackrel{w}{\rightarrow} \Gamma$. It is easy to see that $\Gamma$ is separated because $\Lambda$ is, and that the measures $\mu_{n}:=\sum_{\lambda \in \Lambda} \delta_{\lambda+x_{n}}$ are in $W_{0}^{*}$ and converge to $\mu:=\sum_{\gamma \in \Gamma} \delta_{\gamma}$ in the $\sigma\left(W_{0}^{*}(\mathbb{R}), W_{0}(\mathbb{R})\right)$ topology. (Here it is important that $\Lambda$ is separated; See Remark 7.2.) Let $\varepsilon \in(0,1 / 2)$ and $\eta \in C^{\infty}(\mathbb{R})$ be such that $0 \leq \eta \leq 1$, supp $(\eta) \subseteq[-1,1]$ and $\eta \equiv 1$ on $[-1+\varepsilon, 1-\varepsilon]$.

There is nothing to prove if $D^{-}(\Lambda)=0$. If $D^{-}(\Lambda)>0$, choose $\rho \in \mathbb{R}$ such that $0<\rho<D^{-}(\Lambda)$. Then there exists $r_{0}>0$ such that for all $r \geq r_{0}$ and all $x \in \mathbb{R}$ we have $\#(\Lambda \cap[x-r, x+r]) \geq 2 r \rho$. For $x \in \mathbb{R}$ and $r \geq 2 r_{0}$,

$$
\begin{aligned}
\#(\Gamma \cap[x-r, x+r]) & \geq \int_{\mathbb{R}} \eta\left(\frac{y-x}{r}\right) d \mu(y)=\lim _{n \rightarrow \infty} \int_{\mathbb{R}} \eta\left(\frac{y-x}{r}\right) d \mu_{n}(y) \\
& \geq \liminf _{n \rightarrow \infty} \#\left(\left(\Lambda+x_{n}\right) \cap[x-r(1-\varepsilon), x+r(1-\varepsilon)]\right) \\
& =\liminf _{n \rightarrow \infty} \#\left(\Lambda \cap\left[x-x_{n}-r(1-\varepsilon), x-x_{n}+r(1-\varepsilon)\right]\right) \\
& \geq 2 r \rho(1-\varepsilon) .
\end{aligned}
$$

Hence $D^{-}(\Gamma) \geq \rho(1-\varepsilon)$. As this holds for arbitrary $\varepsilon>0$ and $\rho<D^{-}(\Lambda)$, we conclude that $D^{-}(\Gamma) \geq D^{-}(\Lambda)$.

Remark 7.2 Lemma 7.1 is false for non-separated sets $\Lambda$. For example, if $\Lambda=\left\{k, k+2^{-|k|}: k \in \mathbb{Z}\right\}$, then $D^{-}(\Lambda)=2$, but $\Lambda-n \stackrel{w}{\rightarrow} \mathbb{Z}$, as $n \longrightarrow \infty$ with $n \in \mathbb{N}$, and $D^{-}(\mathbb{Z})=1$. In this case, the measure $\mu$ in the proof is $\mu=\sum_{k \in \mathbb{Z}} 2 \delta_{k}$.

\subsection{Gabor and sampling: postponed proofs}

\section{Proofs of Theorems 2.3 and 2.5}

\section{Step 1: Relation between the relevant operators}

The spanning properties of the Gabor system $\mathcal{G}(g,(-\Lambda) \times \mathbb{Z})$ on $L^{2}(\mathbb{R})$ are encoded in the spectrum of the synthesis operator

$$
\begin{aligned}
& C: \ell^{2}(\Lambda \times \mathbb{Z}) \longrightarrow L^{2}(\mathbb{R}) \\
& \left(c_{\lambda, k}\right)_{\lambda \in \Lambda, k \in \mathbb{Z}} \mapsto \sum_{\lambda \in \Lambda} \sum_{k \in \mathbb{Z}} c_{\lambda, k} e^{2 \pi i k \cdot} g(\cdot+\lambda) .
\end{aligned}
$$

Indeed, $\mathcal{G}(g,(-\Lambda) \times \mathbb{Z})$ is a Riesz sequence if and only if $C$ is bounded below, and $\mathcal{G}(g,(-\Lambda) \times \mathbb{Z})$ is a frame if and only if $C$ is surjective, if and only if $C^{*}$ is bounded below. 
Similarly, the properties of the sets $\Lambda+x$ as sampling and interpolating sets for $V^{2}(g)$ are determined by the operators $P_{\Lambda+x}(g): \ell^{2}(\mathbb{Z}) \rightarrow \ell^{2}(\Lambda)$ represented by the matrices $P_{\Lambda+x}(g)=(g(x+\lambda-k))_{\lambda \in \Lambda, k \in \mathbb{Z}}$, or, equivalently, by the spectral properties of their Banach adjoints

$$
\begin{aligned}
P_{\Lambda+x}(g)^{\prime}: \ell^{2}(\Lambda) & \longrightarrow \ell^{2}(\mathbb{Z}) \\
\left(c_{\lambda}\right)_{\lambda \in \Lambda} & \mapsto \sum_{\lambda \in \Lambda} c_{\lambda} g(x+\lambda-k) .
\end{aligned}
$$

More precisely, according to Lemma 2.2, $\Lambda+x$ is a sampling set for $V^{2}(g)$ if and only if $P_{\Lambda+x}(g)$ is bounded below and it is an interpolating set if and only if $P_{\Lambda+x}(g)^{\prime}$ is bounded below.

It remains to clarify the relation between the operators $C$ and $P_{\Lambda+x}(g)$. We consider the following unitary maps between Hilbert spaces. Let $I=[0,1)$ and $U: \ell^{2}(\Lambda \times \mathbb{Z}) \rightarrow L^{2}\left(I, \ell^{2}(\Lambda)\right)$ be given by $U\left(\left(a_{\lambda, k}\right)_{(\lambda, k) \in \Lambda \times \mathbb{Z})=}\right.$ $\left(m_{\lambda}(x)\right)_{\lambda \in \Lambda}$ with $m_{\lambda}(x)=\sum_{k \in \mathbb{Z}} a_{\lambda, k} e^{2 \pi i k x}$ and $V: L^{2}(\mathbb{R}) \rightarrow L^{2}\left(I, \ell^{2}(\mathbb{Z})\right)$ be given by $\left.V f(x)=(f(x-k))_{k \in \mathbb{Z}}\right)$ for $x \in I$. Now consider the map

$$
\begin{aligned}
\widehat{C}: L^{2}\left(I, \ell^{2}(\Lambda)\right) & \longrightarrow L^{2}\left(I, \ell^{2}(\mathbb{Z})\right) \\
\widehat{C}\left(x \mapsto\left(m_{\lambda}(x)\right)_{\lambda \in \Lambda}\right) & =\left(x \mapsto\left(\sum_{\lambda \in \Lambda} m_{\lambda}(x) g(x+\lambda-k)\right)_{k \in \mathbb{Z}}\right) .
\end{aligned}
$$

In technical jargon, $\widehat{C}$ is the direct integral

$$
\widehat{C}=\int_{I}^{\oplus} P_{\Lambda+x}(g)^{\prime} d x .
$$

These definitions imply that

$$
V C=\widehat{C} U,
$$

and thus $C$ and $\widehat{C}$ have the same spectral properties. It is a standard fact about direct integrals that $\widehat{C}$ is bounded below if and only if ess $\inf _{x} \sigma_{\min }\left(P_{\Lambda+x}(g)^{\prime}\right)>$ 0 - where $\sigma_{\min }(T):=\inf \{\|T x\|:\|x\|=1\}$. Similarly, $\widehat{C}^{*}$ is bounded below if and only if ess inf $\sigma_{x} \sigma_{\min }\left(P_{\Lambda+x}(\bar{g})\right)=\operatorname{essinf}_{x} \sigma_{\min }\left(P_{\Lambda+x}(g)\right)>0$ (see [17, Chapter 1, Part II] for a general reference on direct integrals or [26,35] for direct computations in related contexts).

Step 2: Conclusions. We only argue for Theorem 2.3, Theorem 2.5 follows in the same manner.

Assumption (c) says that $P_{\Lambda+x}(g)$ is bounded below with uniform constants. The direct integral decomposition (7.1) now implies the equivalence between (a) and (c). The implication (b) $\Rightarrow$ (c) follows from the fact that the map $x \mapsto P_{\Lambda+x}(g)$ is continuous from $\mathbb{R}$ to $\mathcal{B}\left(\ell^{2}(\mathbb{Z}), \ell^{2}(\Lambda)\right)$ with respect to the 
operator norm. The continuity is a special case of various jitter error bounds in the sampling literature, see e.g. [20, Lemma 2.2 and Theorem 2.3], and holds precisely when $g \in W_{0}$. Finally, the equivalence of (c) and (d) is a straightforward application of Parseval's identity with $h(\xi)=\sum_{k \in \mathbb{Z}} c_{k} e^{2 \pi i k \xi}$ and the definition of the Zak transform.

Remark 7.3 Common signal processing proofs of Theorems 2.3 and 2.5 for the uniform case $\Lambda=\alpha \mathbb{Z}$ use the Poisson summation formula and are therefore not applicable to non-lattice sets $\Lambda$. The fact that these theorems are true in the stated generality seems to have gone so far unnoticed.

Proof of Theorem 4.3 Without loss of generality we consider the standard Gaussian $\varphi(x):=e^{-\pi x^{2}}$. The general case $e^{-c x^{2}}$ follows then by applying the following change of variables: $f(x) \mapsto f(a x), \Omega \mapsto\left[\begin{array}{cc}a^{-1} & 0 \\ 0 & a\end{array}\right] \cdot \Omega, a=\sqrt{c / \pi}$, which preserves the density of $\Omega$.

In order to show that $\mathcal{G}(\varphi, \Omega)$ spans a dense subspace of $W_{0}$, we show that the only linear functional on $W_{0}$ that annihilates $\mathcal{G}(\varphi, \Omega)$ is the zero functional. In the following

$$
V_{\varphi} \mu(x, \xi):=\int_{\mathbb{R}} \varphi(t-x) e^{-2 \pi i \xi t} d \mu(t) \quad(x, \xi) \in \mathbb{R}^{2}
$$

denotes the short-time Fourier transform of a (Radon) measure $\mu$ (with respect to the Gaussian window $\varphi$ ).

Now suppose that $\mu \in W_{0}^{*}$ is a measure such that $V_{\varphi} \mu\left(v_{1}, v_{2}\right)=0$ for all $\left(v_{1}, v_{2}\right) \in \Omega$. We want to show that $\mu \equiv 0$. By the properties of the shorttime Fourier transform $V_{\varphi} \mu$ is bounded for $\mu \in W_{0}^{*}$ and satisfies the identity $V_{\varphi} \mu(x,-\xi)=F(z) e^{-\pi i x \xi} e^{-\frac{\pi}{2}|z|^{2}}$, where $z=x+i \xi \in \mathbb{C}$ and $F$ is the Bargmann transform of $\mu$. Hence $F$ is analytic and satisfies

$$
\|F\|_{\mathcal{F}^{\infty}}:=\sup _{z \in \mathbb{C}}\left|F(z) e^{-\frac{\pi}{2}|z|^{2}}\right|<\infty
$$

Moreover, $F \equiv 0$ if and only if $\mu=0$. (See for example [6,21], or [23, Section 3.4].)

With these identifications, we reduce the problem to the following. Let $F$ : $\mathbb{C} \rightarrow \mathbb{C}$ be an analytic function such that $F \equiv 0$ on $\bar{\Omega}=\{x-i \xi:(x, \xi) \in \Omega\}$ and $\|F\|_{\mathcal{F} \infty}<\infty$. We want to show that $F \equiv 0$. Suppose not. We can assume that $\|F\|_{\mathcal{F} \infty}=1$ and that $F(0) \neq 0$. (If $F(0)=0$, consider $F(z) / z^{k}$ for a suitable power and normalize.) Then $F: \mathbb{C} \rightarrow \mathbb{C}$ is an entire function that satisfies

$$
|F(z)| \leq e^{\frac{\pi}{2}|z|^{2}}
$$


Let $N(r)$ be the number of zeros of $F$ inside the open disk $D_{r}$ counted with multiplicities. By Jensen's formula,

$$
\int_{0}^{r} \frac{N(t)}{t} d t=\frac{1}{2 \pi} \int_{0}^{2 \pi} \log \left|F\left(r e^{i \theta}\right)\right| d \theta-\log |F(0)| .
$$

By (7.3), $\log \left|F\left(r e^{i \theta}\right)\right| \leq \frac{\pi}{2} r^{2}$. Therefore,

$$
\int_{0}^{r} \frac{N(t)}{t} d t \leq \frac{\pi}{2} r^{2}-\log |F(0)| .
$$

On the other hand, since $D^{-}(\bar{\Omega})=D^{-}(\Omega)>1$, we can choose $v>1$ and $r_{0}>0$ such that, for $r \geq r_{0}, N(r) \geq v \pi r^{2}$. As a consequence,

$$
\int_{0}^{r} \frac{N(t)}{t} d t \geq v \frac{\pi}{2} r^{2}-C
$$

for some constant $C>0$. Since $v>1$, this contradicts (7.4) for $r \gg 0$, and hence $F \equiv 0$, as desired.

Remark 7.4 The Proof of Theorem 4.3 actually shows that $\mathcal{G}(g, \Omega)$ spans a dense subspace of the space $M^{1}(\mathbb{R})$, which consists of functions with integrable short-time Fourier transform. $M^{1}(\mathbb{R})$ embeds continuously into $W_{0}(\mathbb{R})$.

\subsection{Beurling-type characterization of sampling: postponed proof}

Proof of Theorem 3.1 $(a) \Leftrightarrow(b)$ We consider the matrix $P_{\Lambda}(g)=(g(\lambda-$ $k))_{\lambda \in \Lambda, k \in \mathbb{Z}}$ as an operator $\ell^{p}(\mathbb{Z}) \rightarrow \ell^{p}(\Lambda)$. To show that $(a) \Leftrightarrow(b)$, we invoke the following non-commutative version of Wiener's Lemma taken from [28, Proposition 8.1]:

Proposition 7.5 Let $\Lambda$ and $\Gamma$ be relatively separated subsets of $\mathbb{R}$ and $A \in$ $\mathbb{C}^{\Lambda \times \Gamma}$ be a matrix such that

$$
\left|A_{\lambda, \gamma}\right| \leq \Theta(\lambda-\gamma) \quad \lambda \in \Lambda, \gamma \in \Gamma, \text { for some } \Theta \in W_{0} .
$$

Then $A$ is bounded below on some $\ell^{p_{0}}(\Gamma), 1 \leq p_{0} \leq \infty$, i.e., $\|A c\|_{p_{0}} \geq$ $C_{0}\|c\|_{p_{0}}$ for all $c \in \ell^{p_{0}}(\Gamma)$, if and only if $A$ is bounded below on all $\ell^{p}(\Gamma)$, $1 \leq p \leq \infty$.

Since $g \in W_{0}$, the envelope for $P_{\Lambda}(g)$ can be chosen to be $\Theta(x)=|g(x)|$. Then $P_{\Lambda}(g)$ is bounded below on some $\ell^{p_{0}}$, if and only if it is bounded below on all $\ell^{p}$. This gives the desired conclusion. 
The statement in [28] actually covers more general matrices that are concentrated away from a collection of lines; with the notation of [28, Proposition A.1] we apply it with $G=\{1\}$. For related versions of Wiener's Lemma we refer to Sjöstrand's fundamental work [58] and $[1,7,59]$.

$(b) \Rightarrow(c)$ Suppose that $\Lambda$ is a sampling set for $V^{\infty}(g)$ and let $\Gamma \in W_{\mathbb{Z}}(\Lambda)$, so that there exist $k_{n} \in \mathbb{Z}$ such that $\Lambda-k_{n} \stackrel{w}{\rightarrow} \Gamma$. Since $\Lambda$ is a sampling set, the operator $P_{\Lambda}(g): \ell^{\infty}(\mathbb{Z}) \rightarrow \ell^{\infty}(\Lambda)$ is bounded below, so its (pre) adjoint $P_{\Lambda}(g)^{\prime}: \ell^{1}(\Lambda) \rightarrow \ell^{1}(\mathbb{Z})$, represented by the matrix

$$
P_{\Lambda}(g)^{\prime}=(g(\lambda-k))_{k \in \mathbb{Z}, \lambda \in \Lambda},
$$

is onto by the closed range theorem. This means that for every sequence $c \in$ $\ell^{1}(\mathbb{Z})$ there exists an $a \in \ell^{1}(\Lambda)$ such that

$$
c_{k}=\sum_{\lambda \in \Lambda} a_{\lambda} g(\lambda-k), \quad k \in \mathbb{Z},
$$

and $\|a\|_{1} \lesssim\|c\|_{1}$. For $c \in \ell^{1}(\mathbb{Z})$ fixed, we apply this observation to every sequence $\left(c_{k-k_{n}}\right)_{k \in \mathbb{Z}}$ and find a corresponding sequence $a^{n} \in \ell^{1}(\Lambda)$ with $\left\|a^{n}\right\|_{1} \lesssim\|c\|_{1}$ and

$$
c_{k-k_{n}}=\sum_{\lambda \in \Lambda} a_{\lambda}^{n} g(\lambda-k), \quad k \in \mathbb{Z}
$$

Consider the shifted sequence $b^{n} \in \ell^{1}\left(\Lambda-k_{n}\right)$ defined by $b_{\lambda-k_{n}}^{n}=a_{\lambda}^{n}$. We can rewrite (7.6) as

$$
c_{k}=\sum_{\nu \in \Lambda-k_{n}} b_{\nu}^{n} g(v-k), \quad k \in \mathbb{Z}
$$

Consider the measure $\mu_{n}:=\sum_{v \in \Lambda-k_{n}} b_{v}^{n} \delta_{v}$. Then $\mu_{n}$ is a bounded measure, i.e., $\mu \in C_{0}^{*}(\mathbb{R})$, and $\left\|\mu_{n}\right\|_{C_{0}^{*}(\mathbb{R})}=\left\|b^{n}\right\|_{1}=\left\|a^{n}\right\|_{1} \lesssim\|c\|_{1}$. By passing to a subsequence, we may choose a measure $\mu \in C_{0}^{*}(\mathbb{R})$ such that $\mu_{n} \longrightarrow \mu$ in the weak* topology $\sigma\left(C_{0}^{*}(\mathbb{R}), C_{0}(\mathbb{R})\right)$. Since $\Lambda-k_{n} \stackrel{w}{\rightarrow} \Gamma$, it follows that $\Gamma$ is relatively separated and $\operatorname{supp}(\mu) \subseteq \Gamma$ (see [28, Lemmas 4.3 and 4.5]). Hence $\mu=\sum_{\gamma \in \Gamma} b_{\gamma} \delta_{\gamma}$, for some sequence $b$. Moreover $\|b\|_{1}=\|\mu\|_{C_{0}^{*}(\mathbb{R})} \lesssim$ $\liminf _{n}\left\|\mu_{n}\right\|_{C_{0}^{*}(\mathbb{R})} \lesssim 1$. Finally, for each $k \in \mathbb{Z}$,

$$
\begin{aligned}
\sum_{\gamma \in \Gamma} b_{\gamma} g(\gamma-k) & =\int_{\mathbb{R}} g(x-k) d \mu(x)=\lim _{n} \int_{\mathbb{R}} g(x-k) d \mu_{n}(x) \\
& =\lim _{n} \sum_{\nu \in \Lambda-k_{n}} b_{\nu}^{n} g(\nu-k)=c_{k} .
\end{aligned}
$$


Since $c \in \ell^{1}(\mathbb{Z})$ was arbitrary, this shows that $P_{\Gamma}(g)^{\prime}: \ell^{1}(\Gamma) \rightarrow \ell^{1}(\mathbb{Z})$ is onto and therefore $P_{\Gamma}(g): \ell^{\infty}(\mathbb{Z}) \rightarrow \ell^{\infty}(\Gamma)$ is bounded below. Hence $\Gamma$ is a sampling set for $V^{\infty}(g)$.

The implication $(c) \Rightarrow(d)$ is obvious, the equivalence $(d) \Leftrightarrow(e)$ is just language, and the equivalence $(e) \Leftrightarrow(f)$ is standard functional analysis.

$(d) \Rightarrow(b)$ Assume on the contrary that $\Lambda$ is not a sampling set for $p=\infty$. Then there exist sequences $c^{n} \in \ell^{\infty}(\mathbb{Z})$ with $\left\|c^{n}\right\|_{\infty}=1$ such that

$$
\sup _{\lambda \in \Lambda}\left|\sum_{k \in \mathbb{Z}} c_{k}^{n} g(\lambda-k)\right| \rightarrow 0, \quad \text { as } n \rightarrow \infty
$$

Since $\left\|c^{n}\right\|_{\infty}=1$, there exist $k_{n} \in \mathbb{Z}$ such that $\left|c_{k_{n}}^{n}\right| \geq 1 / 2$. Let $d^{n} \in \ell^{\infty}(\mathbb{Z})$ be the shifted sequence $d_{k}^{n}:=c_{k+k_{n}}^{n}$. By passing to a subsequence we may assume that (i) $d^{n} \rightarrow d \in \ell^{\infty}(\mathbb{Z})$ in the $\sigma\left(\ell^{\infty}, \ell^{1}\right)$ topology, and (ii) $\Lambda-k_{n} \stackrel{w}{\rightarrow} \Gamma$ for some (relatively separated) set $\Gamma \in W_{\mathbb{Z}}(\Lambda)$ (see [28, Lemma 4.5]). Let $f:=\sum_{k \in \mathbb{Z}} d_{k} g(\cdot-k) \in V^{\infty}(g)$. Since $c_{k_{n}}^{n}=d_{0}^{n} \longrightarrow d_{0}$, we must have $d_{0} \neq 0$, and therefore $f \not \equiv 0$.

We next show that $f \equiv 0$ on $\Gamma$. Given $\gamma \in \Gamma$, there exists a sequence $\left\{\lambda_{n}: n \geq 1\right\} \subseteq \Lambda$ such that $\lambda_{n}-k_{n} \longrightarrow \gamma$. Since $g \in W_{0}$, it follows that $\left(g\left(\lambda_{n}-k_{n}-k\right)\right)_{k \in \mathbb{Z}} \longrightarrow(g(\gamma-k))_{k \in \mathbb{Z}}$ in $\ell^{1}(\mathbb{Z})$. Since $d^{n} \longrightarrow d$ in $\sigma\left(\ell^{\infty}, \ell^{1}\right)$, we conclude from (7.8) that

$$
\begin{aligned}
|f(\gamma)| & =\left|\sum_{k \in \mathbb{Z}} d_{k} g(\gamma-k)\right|=\lim _{n}\left|\sum_{k \in \mathbb{Z}} d_{k}^{n} g\left(\lambda_{n}-k_{n}-k\right)\right| \\
& =\lim _{n}\left|\sum_{k \in \mathbb{Z}} c_{k}^{n} g\left(\lambda_{n}-k\right)\right|=0 .
\end{aligned}
$$

Hence $f \equiv 0$ on $\Gamma$, which contradicts (d). This completes the proof.

Acknowledgements Open access funding provided by University of Vienna.

Open Access This article is distributed under the terms of the Creative Commons Attribution 4.0 International License (http://creativecommons.org/licenses/by/4.0/), which permits unrestricted use, distribution, and reproduction in any medium, provided you give appropriate credit to the original author(s) and the source, provide a link to the Creative Commons license, and indicate if changes were made.

\section{References}

1. Aldroubi, A., Baskakov, A.G., Krishtal, I.A.: Slanted matrices, Banach frames, and sampling. J. Funct. Anal. 255, 1667-1691 (2008)

2. Aldroubi, A., Gröchenig, K.: Beurling-Landau-type theorems for non-uniform sampling in shift invariant spline spaces. J. Fourier Anal. Appl. 6(1), 93-103 (2000) 
3. Aldroubi, A., Gröchenig, K.: Nonuniform sampling and reconstruction in shift-invariant spaces. SIAM Rev. 43(4), 585-620 (2001)

4. Ascensi, G., Feichtinger, H.G., Kaiblinger, N.: Dilation of the Weyl symbol and BalianLow theorem. Trans. Am. Math. Soc. 366(7), 3865-3880 (2014)

5. Balan, R., Casazza, P.G., Heil, C., Landau, Z.: Density, overcompleteness, and localization of frames. I. Theory. J. Fourier Anal. Appl. 12(2), 105-143 (2006)

6. Bargmann, V.: On a Hilbert space of analytic functions and an associated integral transform. II: a family of related function spaces. Application to distribution theory. Commun. Pure Appl. Math. 14, 187-214 (1961)

7. Baskakov, A.G.: Wiener's theorem and asymptotic estimates for elements of inverse matrices. Funktsional. Anal. i Prilozhen. 24(3), 64-65 (1990)

8. Baxter, B.J.C., Sivakumar, N.: On shifted cardinal interpolation by Gaussians and multiquadrics. J. Approx. Theory 87, 36-59 (1996)

9. Benedetto, J.J., Heil, C., Walnut, D.F.: Differentiation and the Balian-Low theorem. J. Fourier Anal. Appl. 1(4), 355-402 (1995)

10. Beurling, A.: Local harmonic analysis with some applications to differential operators. In: Some Recent Advances in the Basic Sciences, vol. 1 (Proceedings of Annual Science Conference, Belfer Graduate School of Science, Yeshiva University, 1962-1964), pp. 109125. Belfer Graduate School of Science, Yeshiva University, New York (1966)

11. Beurling, A.: Contemporary mathematicians. In: Carleson, L., Malliavin, P., Neuberger, J., Wermer, J. (eds.) The Collected Works of Arne Beurling: Complex Analysis, vol. 1. Birkhäuser Boston Inc, Boston (1989)

12. Blu, T., Marziliano, P., Vetterli, M.: Sampling signals with finite rate of innovation. IEEE Trans. Signal Process. 50(6), 1417-1428 (2002)

13. Boas Jr., R.P.: Entire Functions. Academic Press, New York (1954)

14. Brekke, S., Seip, K.: Density theorems for sampling and interpolation in the BargmannFock space. III. Math. Scand. 73(1), 112-126 (1993)

15. Daubechies, I.: The wavelet transform, time-frequency localization and signal analysis. IEEE Trans. Inf. Theory 36(5), 961-1005 (1990)

16. Daubechies, I., Grossmann, A.: Frames in the Bargmann Hilbert space of entire functions. Commun. Pure Appl. Math. 41(2), 151-164 (1988)

17. Dixmier, J.: Von Neumann Algebras, volume 27 of North-Holland Mathematical Library. North-Holland Publishing Co., Amsterdam (1981). With a preface by E. C. Lance, Translated from the second French edition by F. Jellett

18. Duffin, R.J., Schaeffer, A.C.: A class of nonharmonic Fourier series. Trans. Amer. Math. Soc. 72, 341-366 (1952)

19. Feichtinger, H.G.: Banach convolution algebras of Wiener type. In: Functions, Series, Operators, vol. I, II (Budapest, 1980), pp. 509-524. Coll. Math. Soc. Janos Bolyai. NorthHolland, Amsterdam (1983)

20. Feichtinger, H.G., Molter, U.M., Romero, J.L.: Perturbation techniques in irregular splinetype spaces. Int. J. Wavelets Multiresolut. Inf. Process. 6(2), 249-277 (2008)

21. Folland, G.B.: Harmonic Analysis in Phase Space. Princeton Univ. Press, Princeton (1989)

22. Führ, H., Gröchenig, K., Haimi, A., Klotz, A., Romero, J.L.: Density of sampling and interpolation in reproducing kernel Hilbert spaces. J. London Math. Soc. (to appear). Preprint arXiv: 1607.07803

23. Gröchenig, K.: Foundations of Time-Frequency Analysis. Birkhäuser Boston Inc., Boston (2001)

24. Gröchenig, K.: Localization of frames, Banach frames, and the invertibility of the frame operator. J. Fourier Anal. Appl. 10(2), 105-132 (2004)

25. Gröchenig, K.: Gabor frames without inequalities. Int. Math. Res. Not. IMRN (23), Art. ID rnm111, 21 (2007)

26. Gröchenig, K.: The mystery of Gabor frames. J. Fourier Anal. Appl. 20(4), 865-895 (2014) 
27. Gröchenig, K., Lyubarskii, Y.: Gabor (super)frames with Hermite functions. Math. Ann. 345(2), 267-286 (2009)

28. Gröchenig, K., Ortega Cerdà, J., Romero, J.L.: Deformation of Gabor systems. Adv. Math. 277(4), 388-425 (2015)

29. Gröchenig, K., Stöckler, J.: Gabor frames and totally positive functions. Duke Math. J. 162, 1003-1031 (2013)

30. Hangelbroek, T., Madych, W., Narcowich, F., Ward, J.D.: Cardinal interpolation with Gaussian kernels. J. Fourier Anal. Appl. 18(1), 67-86 (2012)

31. Heil, C.: An introduction to weighted Wiener amalgams. In: Krishna, M., Radha, R., Thangavelu, S. (eds.) Wavelets and Their Applications (Chennai. January 2002), pp. 183216. Allied Publishers, New Delhi (2003)

32. Heil, C.: History and evolution of the density theorem for Gabor frames. J. Fourier Anal. Appl. 13(2), 113-166 (2007)

33. Janssen, A.J.E.M.: The Zak transform and sampling theorems for wavelet subspaces. IEEE Trans. Signal Process. 41, 3360-3364 (1993)

34. Janssen, A.J.E.M.: Signal analytic proofs of two basic results on lattice expansions. Appl. Comput. Harmon. Anal. 1(4), 350-354 (1994)

35. Janssen, A.J.E.M.: Duality and biorthogonality for Weyl-Heisenberg frames. J. Fourier Anal. Appl. 1(4), 403-436 (1995)

36. Janssen, A.J.E.M.: Some Weyl-Heisenberg frame bound calculations. Indag. Math. 7, 165$182(1996)$

37. Janssen, A.J.E.M.: On generating tight Gabor frames at critical density. J. Fourier Anal. Appl. 9(2), 175-214 (2003)

38. Janssen, A.J.E.M., Strohmer, T.: Hyperbolic secants yield Gabor frames. Appl. Comput. Harmon. Anal. 12(2), 259-267 (2002)

39. Jia, R.Q., Micchelli, C.A.: Using the refinement equations for the construction of prewavelets II: powers of two. In: Laurent, P.J., Le Méhauté, A., Schumaker, L.L. (eds.) Curves and Surfaces, pp. 209-246. Academic Press, New York (1991)

40. Karlin, S.: Total Positivity, vol. I. Stanford University Press, Stanford (1968)

41. Kloos, T., Stöckler, J.: Zak transforms and Gabor frames of totally positive functions and exponential B-splines. J. Approx. Theory 184, 209-237 (2014)

42. Kozma, G., Nitzan, S.: Combining Riesz bases. Invent. Math. 199(1), 267-285 (2015)

43. Landau, H.J.: Necessary density conditions for sampling and interpolation of certain entire functions. Acta Math. 117, 37-52 (1967)

44. Lemvig, J.: On some Hermite series identities and their applications to Gabor analysis. Monatsh. Math. 182, 899-912 (2017)

45. Lev, N.: Riesz bases of exponentials on multiband spectra. Proc. Am. Math. Soc. 140(9), 3127-3132 (2012)

46. Levin, B.Y.: Lectures on Entire Functions. American Mathematical Society, Providence, RI, (1996). In collaboration with and with a preface by Yu. Lyubarskii, M. Sodin and V. Tkachenko, Translated from the Russian manuscript by Tkachenko

47. Lyubarskiı̆, Y.I.: Frames in the Bargmann space of entire functions. In: Entire and Subharmonic Functions, pp. 167-180. Advances in Soviet Mathematics 11. American Mathematical Society, Providence (1992)

48. Narcowich, F.J., Ward, J.D., Wendland, H.: Sobolev bounds on functions with scattered zeros, with applications to radial basis function surface fitting. Math. Comput. 74, 743-763 (2005)

49. Ortega-Cerdà, J., Seip, K.: Fourier frames. Ann. Math. (2) 155(3), 789-806 (2002)

50. Ron, A.: Introduction to shift-invariant spaces. Linear independence. In: Dyn, N., Leviatan, D., Levin, D., Pinkus, A. (eds.) Multivariate Approximation and Applications, pp. 152-211. Cambrigde University Press, Cambridge (2001) 
51. Ron, A., Shen, Z.: Weyl-Heisenberg frames and Riesz bases in $L_{2}\left(\mathbb{R}^{d}\right)$. Duke Math. J. 89(2), 237-282 (1997)

52. Schoenberg, I.J.: On totally positive functions, Laplace integrals and entire functions of the Laguerre-Pólya-Schur type. Proc. Natl. Acad. Sci. USA 33, 11-17 (1947)

53. Schoenberg, I.J.: On variation-diminishing integral operators of the convolution type. Proc. Natl. Acad. Sci. USA 34, 164-169 (1948)

54. Schoenberg, I.J.: On Pólya frequency functions, I. The totally positive functions and their Laplace transforms. J. Anal. Math. 1, 331-374 (1951)

55. Schoenberg, I.J., Whitney, A.: On Pólya frequence functions, III. The positivity of translation determinants with an application to the interpolation problem by spline curves. Trans. Amer. Math. Soc. 74, 246-259 (1953)

56. Seip, K.: Density theorems for sampling and interpolation in the Bargmann-Fock space. J. Reine Angew. Math. 429, 91-106 (1992)

57. Seip, K.: Interpolation and Sampling in Spaces of Analytic Functions. University Lecture Series, vol. 33. American Mathematical Society, Providence (2004)

58. Sjöstrand, J.: Wiener type algebras of pseudodifferential operators. Séminaire sur les équations aux Dérivées Partielles, 1994-1995, École Polytech, Palaiseau, Exp. No. IV, 21 (1995)

59. Sun, Q.: Wiener's lemma for infinite matrices. Trans. Amer. Math. Soc. 359(7), 3099-3123 (2007). (electronic)

60. Unser, M.: Sampling—50 years after Shannon. Proc. IEEE 88(4), 569-587 (2000)

61. Walter, G.G.: Wavelets and Other Orthogonal Systems with Applications. CRC Press, Boca Raton (1994) 